\title{
THE NUCLEUS OF C16.16: ANOTHER CASE OF A DOUBLE-PEAKED ACTIVE GALACTIC NUCLEUS
}

\author{
A. Rodríguez-Ardila, ${ }^{1}$ M. G. Pastoriza, ANd Eduardo BicA \\ Departamento de Astronomia, Instituto de Física, Universidade Federal Rio Grande do Sul, \\ C.P. 15051, 91501-970 Porto Alegre, RS, Brazil \\ AND \\ J. MAZA ${ }^{2}$ \\ Departamento de Astronomía, Universidad de Chile, Casilla 36-D, Santiago, Chile \\ Received 1995 April 17; accepted 1995 December 15
}

\begin{abstract}
We study optical spectra in high and low activity states of the faint, distant Seyfert 1 galaxy C16.16. A strong stellar population contribution to the continuum is observed, even in the high-state spectrum. The low-ionization emission lines show large equivalent widths. Narrow-line ratios are used to parameterize and investigate the physical conditions in the narrow-line region. Photoionization models with nitrogen depletion, ionization continuum typical of an active galactic nucleus (AGN), and an ionization parameter $U=10^{-2.8}$ reproduce the observed narrow lines. The AGN continuum shows a strong variation, while the Balmer lines vary moderately. The Balmer lines have a broad and asymmetric component with double-peaked structure. Using the profiles of emission lines originated in a relativistic circular disk, we successfully fit the observed broad $\mathrm{H} \alpha$ profile. This disk component shows no variation in the observations. A variable, intermediate-width $\left(\sim 6000 \mathrm{~km} \mathrm{~s}^{-1}\right)$ Balmer component is also found. These two components seem to arise by different processes.
\end{abstract}

Subject headings: galaxies: individual (C16.16) — galaxies: nuclei - galaxies: Seyfert - line: profiles

\section{INTRODUCTION}

One of the characteristic features of active galactic nuclei (AGNs) is their broad emission lines. In recent years, these lines have been shown to vary in response to changes in the photoionizing continuum (see, e.g., Reichert et al. 1994; Stirpe et al. 1994). The most accepted model for the central engine is a supermassive black hole powered by accretion, and most probably by an accretion disk (Collin-Souffrin 1987). This model could explain the observed broad lines, particularly the Balmer lines (and continuum) and the Fe II multiplets, which would be generated in the inner, weakly ionized part of the disk, while high-ionization lines, e.g., Ly $\alpha$ or C IV, would be produced in a dilute, outflowing medium external to it (see Rokaki, Boisson, \& Collin-Souffrin 1992 and references therein). The best observational evidence for this scenario occurs in objects with double-peaked broad emission lines, although it is not restricted to such objects since single-peaked profiles can also be produced in accretion disks (Dumont \& Collin-Souffrin 1990). Recently, in a survey of moderate-redshift objects (Eracleous \& Halpern 1994), a new "class" of radio-loud objects was argued on the basis of the following properties that disklike emitters seem to possess: (1) average $\mathrm{H} \alpha \mathrm{FWHM}$ on the order of $12,500 \mathrm{~km} \mathrm{~s}^{-1}$, (2) strong starlight contribution around $\mathrm{H} \alpha$, and (3) large equivalent widths of low-ionization forbidden lines. From this survey, it was found that $10 \%$ of a sample of 94 radio galaxies and radio-loud quasars showed doublepeaked $\mathrm{H} \alpha$ profiles that could be fitted by a simple relativistic disk model.

Several AGNs with the above characteristics have been identified during the Calán-Tololo survey (Maza et al. 1989,

\footnotetext{
${ }^{1}$ CNPq Fellow.

2 Visiting Astronomer, Cerro Tololo Inter-American Observatory. CTIO is operated by AURA, Inc., under contract to the National Science Foundation.
}

1992, 1994). In an attempt to provide basic data to improve the global picture of AGNs, we have begun a systematic study of these galaxies from the list of Maza et al. (1989, 1992, 1994), in particular those with Balmer line profiles that suggest disklike structure.

In this paper we present optical spectroscopy of the distant southern galaxy C16.16, cataloged by Maza et al. (1992) as Seyfert 1. This galaxy has $B=17.19$ and a radial velocity $v_{r}=22,864 \mathrm{~km} \mathrm{~s}^{-1}$. The absolute blue magnitude $M_{B}=-20.22$ indicates that this object has a moderate luminosity. Very little (i.e., nothing) is known about the properties of this galaxy at other wavelengths. A search in the IUE and EXOSAT databases shows that this object has not been observed in these spectral regions. Also, there is no available information about it in the radio domain. An inspection of the galaxy's image on the ESO/SERC J plate shows a fuzzy halo, which, however, does not allow one to classify it morphologically.

In $\S 2$, we present the observations and data reduction. The continuum analysis is set out in $\S 3$. In $\S 4$, the narrowline emission spectrum and photoionization models are described. In $\S 5$, the broad-line emission region, variability, and the double-peaked structure of the Balmer lines are discussed. Conclusions are given in $\S 6$. A value of the Hubble constant of $H_{0}=75 \mathrm{~km} \mathrm{~s}^{-1} \mathrm{Mpc}^{-1}$ has been assumed throughout.

\section{OBSERVATIONS AND DATA REDUCTION}

The two spectra of C16.16 were obtained in 1992 October and 1994 August with the $4 \mathrm{~m}$ telescope at Cerro Tololo Inter-American Observatory (CTIO), equipped with a Ritchey-Chrétien spectrograph and a CCD detector. They cover the wavelength range $3200-6850 \AA$ and have a resolution of $\sim 10 \AA$. The observations were made using a slit width of $2^{\prime \prime}$. For flux calibration, three standard stars were observed during each night. An exposure of $\mathrm{He}-\mathrm{Ne}-\mathrm{Ar}$ 


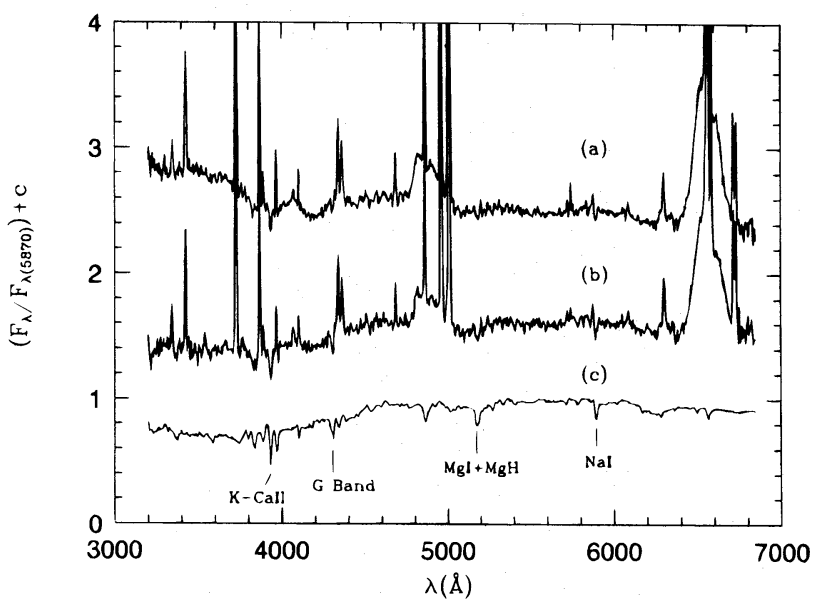

Fig. 1.-Spectra of C16.16 obtained in 1992 October (a) and 1994 August (b) and the resulting stellar population template (c). The spectra are normalized at $\lambda 5870$, and spectra $a$ and $b$ have been shifted by an additive constant for clarity.

lamp was taken after observing the object in order to derive the wavelength calibration. The data reduction was carried out at the CTIO La Serena Computing Center with IRAF. Sky subtraction was performed by using parts of the sky where no flux from the stellar population of the galaxy itself was evident. We have extracted the observed spectra from a region of $2^{\prime \prime} \times 9^{\prime \prime}(\sim 3 \mathrm{kpc} \times \sim 13.5 \mathrm{kpc})$. No reddening correction due to Galactic extinction was needed (see Burstein $\&$ Heiles 1984).

In addition to the absolute flux calibration with the standard stars, a scaling of the spectra was made by assuming that the [O III] $\lambda 5007$ flux does not vary over the timescale of interest. The value we adopted for the absolute flux in [O III] $\lambda 5007$ is $4.6 \times 10^{-14} \mathrm{ergs} \mathrm{s}^{-1} \mathrm{~cm}^{-2}$, which corresponds to that of the first run. Such "internal calibration" may reach an accuracy on the line fluxes of better than $5 \%$ (Stirpe, de Bruyn, \& van Groningen 1988; Peterson et al. 1991). Figure 1 shows the redshift-corrected spectra. In 1992 October, the AGN was in a high activity state (spectrum a), whereas in 1994 August it was in a low one (spectrum b).

\section{CONTINUUM ANALYSIS}

\subsection{The Stellar Contribution}

In order to obtain the pure emission spectrum from the AGN of C16.16, it is necessary to estimate which fraction of the total continuum emission arises from the stellar component (see Fig. 1). The treatment of this continuum was carried out in two steps: (1) identification of the stellar population type and (2) subtraction from the observed spectra. Several stellar population types in the spectral range 3700-9700 §̊ were observed by Bica (1988) in normal spiral and early-type galaxy nuclei. The templates S1-S4 consist of red stellar populations of decreasing average metallicity. S5-S7 form a sequence with increasing contribution from blue components (with ages $t \leq 500 \mathrm{Myr}$ ). In order to identify the appropriate template, we measured, in the high- and low-state spectra (already normalized to the [O III] $\lambda 5007$ line flux), the equivalent widths of the Ca II K, $\mathrm{G}$ band, and $\mathrm{Mg} \mathrm{I}+\mathrm{Mg} h$ absorption features, following the spectral windows and continuum tracings as in Bica \& Alloin (1986). The values obtained are shown in Table 1. Errors in these values are on the order of $7 \%$.
TABLE 1

Equivalent WiDths of the Absorption LINES OBSERVED

\begin{tabular}{ccc}
\hline \hline Line & $\begin{array}{c}W_{\text {high }} \\
(\AA)\end{array}$ & $\begin{array}{c}W_{\text {low }} \\
(\AA)\end{array}$ \\
\hline $\mathrm{Ca}$ II K $\ldots \ldots \ldots \ldots \ldots$. & 5.4 & 7.6 \\
$\mathrm{G} \mathrm{band} \ldots \ldots \ldots \ldots \ldots$ & 1.1 & 3.0 \\
$\mathrm{Mg} \mathrm{I}+\mathrm{Mg} h \ldots \ldots \ldots$ & 2.1 & 2.3 \\
$I(\lambda 4020) / I(\lambda 5870) \ldots \ldots$ & 1.0 & 0.8 \\
$I(\lambda 4570) / I(\lambda 5870) \ldots \ldots$ & 1.0 & 1.0 \\
\hline
\end{tabular}

In order to constrain the stellar population template, the $W$-values from the low-state spectrum were used. By comparing $W$-values and subtracting different templates at various proportions, we concluded that the $\mathrm{S} 6$ template accounts best for the stellar population of C16.16. The amount of stellar components with ages $t \leq 500 \mathrm{Myr}$ in this template is $24 \%$ of the continuum at $5870 \AA$ (Bica 1988). The S6 template was extended to the near-UV (3200-3800 $\AA$ A) by use of the recent near-UV star cluster library of Bica, Alloin, \& Schmitt (1994). The resulting template in the range 3200-6900 $\AA$ is also shown in Figure 1 (spectrum c).

Finally, we subtracted the S6 template from the two spectra in a proportion that canceled out the absorption lines. This proportion corresponds to $90 \%$ of the continuum at $5870 \AA$ for the low-state spectrum and $70 \%$ for the high-activity spectrum. At $\lambda=3200 \AA$, this fraction is $\sim 85 \%$ and $\sim 34 \%$, respectively, indicating a strong variation of the near-UV continuum of the AGN.

The important stellar population contribution to C16.16's spectrum can be understood in terms of the spatial sampling, which amounts to $\sim 3 \mathrm{kpc} \times \sim 13.5 \mathrm{kpc}$ (see $\S 2$ ). This means that most of the galaxy's body was included in the slit. If one is dealing with a spiral galaxy, the mixture of blue and red stellar populations in \$6 may reflect the bulge and part of the disk components. Otherwise, in the case of an elliptical galaxy, a burst of star formation has necessarily occurred.

\subsection{The Nonstellar Continuum}

After the stellar population subtraction, we analyzed the AGN continuum. We first adjusted a power-law continuum, represented by $F_{\lambda}=k \lambda^{\alpha-2}$, to the entire range of the high-state spectrum. The best fit was obtained for $\alpha=0.85 \pm 0.05$. In the low-state spectrum, this component was reduced to noise level, with $\alpha=2$. The procedure to fit the strong near-UV flux excess (3200-3900 $\AA$ ) present in the high-state spectrum is similar to that followed by Wills, Netzer, \& Wills (1985). They concluded that this excess is due to Balmer continuum (Bac), the merging of high-order Balmer lines, and $\mathrm{Fe}$ II emission. This last component is also present along the optical region, forming a kind of pseudocontinuum. We selected a Balmer continuum model from the grid of Wills et al., obtained under a variety of conditions, adopting as free parameters the cloud thickness, ionization parameter, and cloud density. The best agreement was found for the model with $\tau_{\mathrm{Bac}}=1$ and $T=1.2 \times 10^{4}$ $\mathrm{K}$, which was suitably scaled to match the observed flux density above the power law at $3200 \AA$. A synthetic Fe II model from the grid of Wills et al. was then added to the entire continuum because the general signatures of this ion are clearly present in the spectrum as two bumps centered at 5250 and $4500 \AA$. The parameters for the best matching 


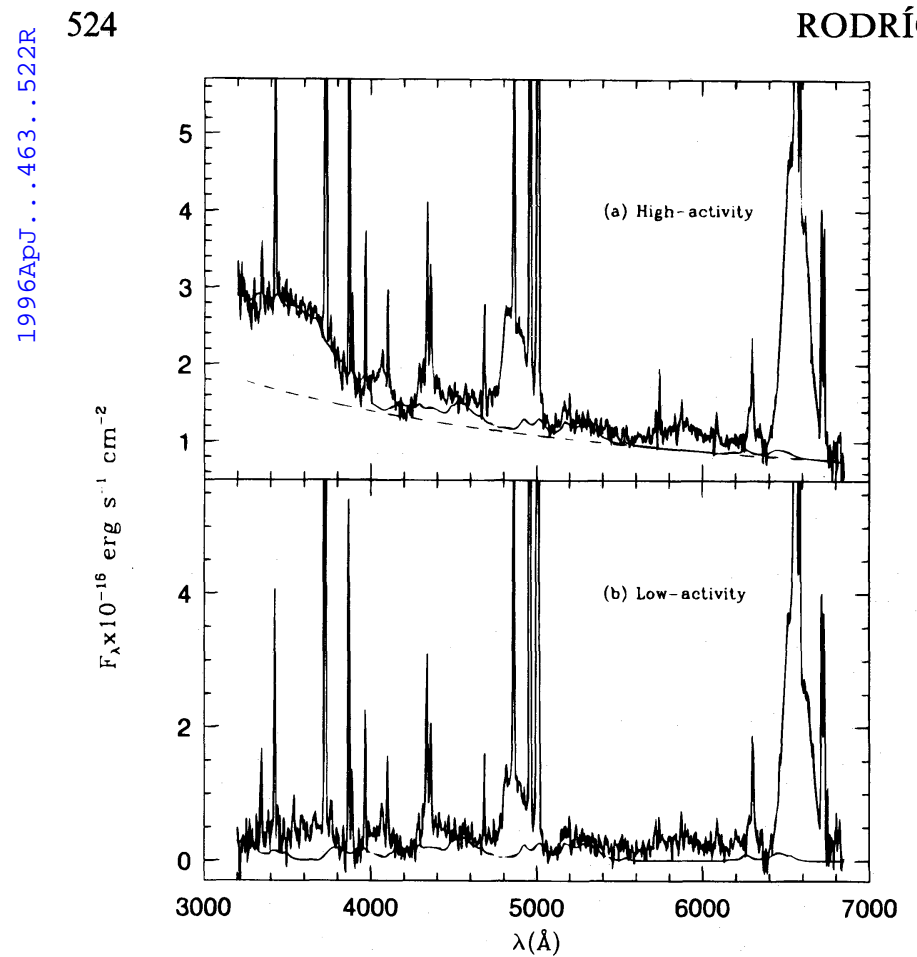

Fig. 2.-Continuum decomposition applied to the C16.16 spectra after subtraction of the underlying stellar population contribution. (a) Highstate spectrum together with the power-law continuum (dashed curve) and the $\mathrm{Fe}$ II emission model plus the Balmer continuum (solid curve). Highorder Balmer lines are included. (b) Low-state spectrum together with the $\mathrm{Fe}$ II model. No Balmer and power-law contribution was found in this spectrum.

model are $\tau_{\mathrm{Bac}}=1.5$ and $v_{t}=3.4 \mathrm{~km} \mathrm{~s}^{-1}$. The remaining flux excess, constrained to the interval $3646-3900 \AA$, corresponds to the blending of higher order Balmer lines, which can easily be accounted for. The total Fe II and Balmer continuum fluxes are given in Table 2 . Figure 2 shows the two spectra after removal of the starlight contribution together with the adjusted continuum model. The relative uncertainty in the continuum fit is on the order of $20 \%$ for the low-state spectrum and $15 \%$ for the high-state one. After subtracting the $\mathrm{Fe}$ II contribution, the [O III] $\lambda 5007 /$ [O III] $\lambda 4959$ ratio was 2.99 for the first case and 3.01 for the second, which ensures the validity of the method for removing this Fe II contribution.

We conclude from this analysis that the variation of the near-UV excess in C16.16 reflects the variation of the highorder Balmer lines and the Balmer continuum due to the variation of the ionizing continuum, the optical extension of which is related to the power law.

\section{THE NARROW EMISSION LINE SPECTRUM}

The total fluxes of the narrow emission line components were measured under the assumption that the lines were well represented by Gaussian profiles. When deblending lines, we have assumed theoretical values for the line ratios [O III] $\lambda 5007 / \lambda 4959$ and [N II] $\lambda 6584 / \lambda 6548$. Prior to measuring the flux of the optical lines, we removed the underlying power law as well as the Balmer continuum and the Fe II contribution by modeling their emission over the entire observed range (see $\S 3.2$ ).

We obtained a reasonable and consistent representation of all narrow-line profiles (with $\chi^{2}<0.5$ for all the lines and less than 0.1 for the majority of them). Table 2 presents the measured line fluxes relative to the narrow $\mathrm{H} \beta$ component
TABLE 2

Emission-Line Parameters

\begin{tabular}{|c|c|c|c|}
\hline Line & $F_{\lambda} / F_{\mathrm{H} \beta}$ & $\begin{array}{c}\text { FWHM } \\
\left(\mathrm{km} \mathrm{s}^{-1}\right)\end{array}$ & $\begin{array}{c}\text { Photoionization } \\
\text { Model }\end{array}$ \\
\hline$[\mathrm{Ne} \mathrm{v}] \lambda 3426$. & 0.7 & 788 & \\
\hline$[\mathrm{O} \mathrm{II}]^{\mathrm{a}} \lambda 3727 \ldots$ & 2.3 & 716 & 4.1 \\
\hline H9 $\lambda 3838 \ldots$. & 0.07 & 717 & \\
\hline$[\mathrm{Ne} \mathrm{III}] \lambda 3870 \ldots$ & 1.0 & 630 & 1.1 \\
\hline H8 + He I $23890 \ldots \ldots \ldots$. & 0.2 & 788 & - \\
\hline $\mathrm{H} \epsilon+[\mathrm{Ne} \quad \mathrm{III}] \lambda 3969 \ldots \ldots$ & 0.5 & 666 & 0.1 \\
\hline$[\mathrm{S} \mathrm{II}]^{\mathrm{b}} \lambda 4071 \ldots \ldots \ldots \ldots \ldots$ & 0.1 & 1030 & \\
\hline $\mathrm{H} \delta^{\mathrm{c}} \lambda 4103$. & 0.3 & 542 & 0.30 \\
\hline $\mathrm{H} \delta^{\mathrm{d}} \lambda 4102 \ldots \ldots \ldots \ldots \ldots$ & 0.4 & 7532 & \\
\hline $\mathrm{H} \gamma^{\mathrm{c}} \lambda 4341 \ldots \ldots \ldots \ldots$ & 0.5 & 542 & 0.50 \\
\hline $\mathrm{H} \gamma^{\mathrm{d}} \lambda 4344 \ldots \ldots$ & 1.6 & 7527 & \\
\hline [O $\mathrm{III}] \lambda 4363 \ldots$ & 0.4 & 521 & 0.05 \\
\hline He II $\lambda 4684 \ldots$ & 0.3 & 654 & 0.2 \\
\hline $\mathrm{H} \beta^{\mathrm{c}} \lambda 4861 \ldots \ldots \ldots \ldots \ldots$ & 1.0 & 545 & 1.0 \\
\hline $\mathbf{H} \beta^{\mathrm{d}, \mathrm{e}} \lambda 4863 \ldots \ldots \ldots \ldots$ & 4.6 & 8900 & $\ldots$ \\
\hline $\mathrm{H} \beta^{\mathrm{d}, \mathrm{f}} \lambda 4863 \ldots \ldots \ldots \ldots \ldots$ & 4.0 & 8820 & \\
\hline$[\mathrm{O} \mathrm{III}] \lambda 5007$. & 10.6 & 521 & 11.2 \\
\hline $\mathrm{He}^{\mathrm{c}} \lambda 5875 \ldots$ & 0.07 & 408 & 0.1 \\
\hline $\mathrm{He} \mathrm{I}^{\mathrm{d}} \lambda 5877 \ldots \ldots \ldots$ & 0.8 & 9800 & $\ldots$ \\
\hline$[\mathrm{Fe}$ VII $] \lambda 6086 \ldots \ldots$ & 0.1 & 540 & \\
\hline$[\mathrm{O} \text { I }]^{\mathrm{c}} \overrightarrow{\lambda 6302 \ldots \ldots \ldots \ldots}$ & 0.2 & 418 & 0.2 \\
\hline$\left[\mathrm{O}_{\mathrm{I}}\right]^{\mathrm{d}} \lambda 6297 \ldots \ldots \ldots \ldots \ldots$ & 0.5 & 2019 & \\
\hline $\mathrm{H} \alpha^{\mathrm{c}} \lambda 6563 \ldots \ldots \ldots \ldots \ldots$ & 2.9 & 378 & 2.7 \\
\hline$H \alpha^{\mathrm{d}, \mathrm{e}} \lambda{ }^{\prime} 6565 \ldots \ldots \ldots \ldots \ldots$ & 14.2 & 9100 & 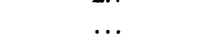 \\
\hline $\mathrm{H} \alpha^{\mathrm{d}, \mathrm{f}} \lambda 6565 \ldots \ldots \ldots$ & 13.0 & 9060 & \\
\hline$[\mathrm{N}$ II $] \lambda 6583 \ldots \ldots \ldots \ldots$ & 1.0 & 396 & 0.9 \\
\hline$[\mathrm{S}$ II $] \lambda 6717 \ldots \ldots \ldots \ldots \ldots$ & 0.7 & 440 & 0.5 \\
\hline$[\mathrm{S}$ II $] \lambda 6731 \ldots \ldots \ldots \ldots \ldots$ & 0.6 & 440 & 0.6 \\
\hline Fe II $(3200-6850 \AA)^{\mathrm{e}} \ldots \ldots$ & 7.9 & $\ldots$ & 0.0 \\
\hline $\mathrm{Fe}$ II $(3200-6850 \AA)^{\mathrm{f}} \ldots \ldots$ & 3.1 & $\ldots$ & $\ldots$ \\
\hline Bac $(3200-3646 \AA)^{8} \ldots \ldots \ldots$ & 12.4 & $\ldots$ & $\ldots$ \\
\hline
\end{tabular}

NoTes.-Wavelengths are given in $\AA ; F_{\mathrm{H} \beta}=4.3 \times 10^{-15} \mathrm{ergs} \mathrm{cm}^{-2}$ $\mathrm{s}^{-1}$.

a Blend of [O II] $\lambda 3726$ and $[\mathrm{O} \mathrm{II}] \lambda 3729$.

b Blend of [S II] $\lambda 4068$ and [S II] $\lambda 4074$.

c Narrow component.

d Broad component.

e In high-state spectrum.

f In low-state spectrum.

8 Balmer continuum.

and the corresponding FWHM. The error in the line fluxes, estimated according to the noise level around each line, was in all cases less than $10 \%$.

We were not able to fit the broad Balmer lines with Gaussian profiles, particularly the broad $\mathrm{H} \beta$ profile, due to their high asymmetry and double-peaked structure. The fluxes for these lines were measured by numerical integration of the line profiles. Their values are also listed in Table 2 .

The narrow-line spectrum, emitted in a relatively low density region (Evans 1988), shows a wide range of ionization by the presence of [O I $]-[\mathrm{Fe}$ VII $]$ lines. The lines [O III] $\lambda \lambda 4959,5007$, as well as [O III] $\lambda 4363$, have broad bases with FWHM $\sim 1800 \mathrm{~km} \mathrm{~s}^{-1}$. This suggests the presence of an intermediate zone (the transition-line region; Evans 1988 ) with density $N_{e} \leq 10^{6.5} \mathrm{~cm}^{-3}$ where the [O III] wings are emitted. Also, the strong [O I] $\lambda 6300$ line suggests the presence of an extended, large, partially ionized zone, which is in agreement with the observation of low-ionization lines with large equivalent widths in objects with double-peaked Balmer lines (Eracleous \& Halpern 1994). The remaining narrow lines were adjusted with a single component with FWHM $\sim 700 \mathrm{~km} \mathrm{~s}^{-1}$ or less.

It is possible to derive some parameters about the condi- 
tions of the narrow-line region (NLR) using the values of fluxes listed in Table 2. From these values, we obtain a Balmer decrement $\mathrm{H} \alpha / \mathrm{H} \beta=2.8$, indicating essentially no internal absorption in the NLR clouds. The electron temperature and density were calculated using the three-levelatom approximation of McCall (1984) by the [O III] $(\lambda 4959+\lambda 5007) / \lambda 4363$ and $[\mathrm{S}$ II $] \lambda 6717 / \lambda 6731$ line ratios. We found values of $T_{e}=10,500_{-700}^{+1500} \mathrm{~K}$ and $N_{e}=510_{-100}^{+150}$ $\mathrm{cm}^{-3}$, respectively. These values cannot be taken as representative of the entire NLR. Undoubtedly, there are wide ranges in $N_{e}$ and $T_{e}$ along this region, but it is significant to estimate an overall mean value for $T_{e}$ and $N_{e}$.

It is also possible to make some crude estimates about the mass and size of the NLR using the expressions in Osterbrock (1989, p. 327). We obtain $M_{\mathrm{NLR}}=150 M_{\odot}$ and $r_{\mathrm{NLR}}=150 \mathrm{pc}$. The assumed covering factor was 0.1 .

The observed emission-line spectrum was compared to photoionization models in order to derive consistent values for the metallicity and the ionization parameter for C16.16's AGN. The calculations were performed using the photoionization code CLOUDY (Ferland 1989). The adopted ionizing continuum was " table AGN," similar to that deduced by Mathews \& Ferland (1987) and which consists of a broken power law of the form $F_{v}=a v^{-\alpha}$ with $\alpha$ varying between 0.5 and 3 depending on the wavelength interval.

Several sequences of models were computed by varying the ionization parameter $U$, the electron density $n_{e}$, and the chemical abundances $z$. In the last column of Table 2 we show the narrow-line emission fluxes relative to $\mathrm{H} \beta$. They were obtained from the best matching model, characterized by $U=10^{-2.8}, n_{e}=10^{3.2} \mathrm{~cm}^{-3}, T_{e}([\mathrm{O} \mathrm{III}])=9100 \mathrm{~K}$, and solar abundances except for nitrogen, which was found to be 0.5 solar. We must be careful about this last result because the observed region $(\sim 3 \mathrm{kpc} \times \sim 13.5 \mathrm{kpc})$ shows evidence of star formation (as deduced from the relatively high percentage of blue stellar population found in the galaxy; see §3.1). The star-forming regions around the nucleus would contaminate the narrow-line emission spectrum, increasing the $\mathrm{H} \alpha /[\mathrm{N} \mathrm{II}]$ line ratio. This effect, however, seems to be negligible because of the high luminosity of the AGN compared to that of the hypothetical regions. Also, the match between the high- and low-activity NLR fluxes indicates that even when the nucleus is in a low state, in which this contamination is expected to be stronger, there is no appreciable increase in the line flux. We therefore regard this nitrogen subabundance as real.

\section{BROAD EMISSION LINES}

The most striking feature in the emission-line spectrum, free of all continuum contribution, is the presence of broad, variable, and asymmetric Balmer lines with peaks at both sides of the lines, significantly displaced in velocity with respect to the host galaxy. Although the first three characteristics are very common in AGNs (Wamsteker et al. 1990; Korista 1992) and have been attributed to a multicomponent broad-line region, the double-peaked structure seen in the $\mathrm{H} \alpha$ and $\mathrm{H} \beta$ lines of C16.16 is very similar to that displayed by a small number of AGNs, most of them classified as radio galaxies (i.e., 3C 382 in Osterbrock, Koski, \& Phillips 1976; Arp 102B in Chen \& Halpern 1989; 3C 390.3 in Eracleous \& Halpern 1994).

\subsection{Line Profile Changes}

As we have pointed out, the nature of the variability of the Seyfert 1 galaxy C16.16 can be deduced from the fact that both the near-UV excess and the power-law spectrum changed dramatically between the two available observations (see Fig. 2). The broad Balmer lines, however, showed a moderate variation, as illustrated for the $\mathrm{H} \alpha$ profile in the upper portion of Figure 3. The varying $\mathrm{H} \alpha$ component, obtained from the difference between these two states of activity, exhibits a rather symmetrical, flat-topped profile with FWHM $\sim 6500 \AA$ (see Fig. 3). We deduce that the bulk of variations occurs in the intermediate width of the Balmer component rather than in the broad wings of the line. The double-peaked structure does not show variability in the two observations. However, variations of this component have been observed in some galaxies that present this structure (e.g., 1E 0450.3 - 1817 in Eracleous \& Halpern 1994; Akn 120 in Alloin, Poisson, \& Pelat 1988). In this case, the absence of variation of the double-peaked profile indicates that it does not respond to the continuum variations with the same time delay as do the near-UV excess and the intermediate Balmer components. It may also indicate that the processes producing these structures are not the same.

\subsection{The Double-peaked Structure}

The interpretation of the double-peaked broad Balmer lines is controversial. Gaskell (1988) took them as evidence for two separate broad-line regions; Chen, Halpern, \& Filippenko (1989) and Chen \& Halpern (1989), as direct kinematical evidence for a relativistic Keplerian disk; and Cassidy \& Raine (1993) proposed a model for the broadline region in which clouds are injected into an outflowing wind from a rotating disk. Another attractive scenario for producing double-peaked profiles is the bipolar flow model (Zheng, Binette, \& Sulentic 1990; Zheng, Veilleux, \& Grandi 1991), in which clouds moving radially outward are confined to a pair of oppositely directed cones whose common axis passes through the origin (i.e., the location of the continuum source).

Assuming that the double-peaked broad $\mathrm{H} \alpha$ line is generated in an accretion disk, we computed several profiles following the simple relativistic Keplerian disk model proposed by Chen \& Halpern (1989) to fit them to this line. The

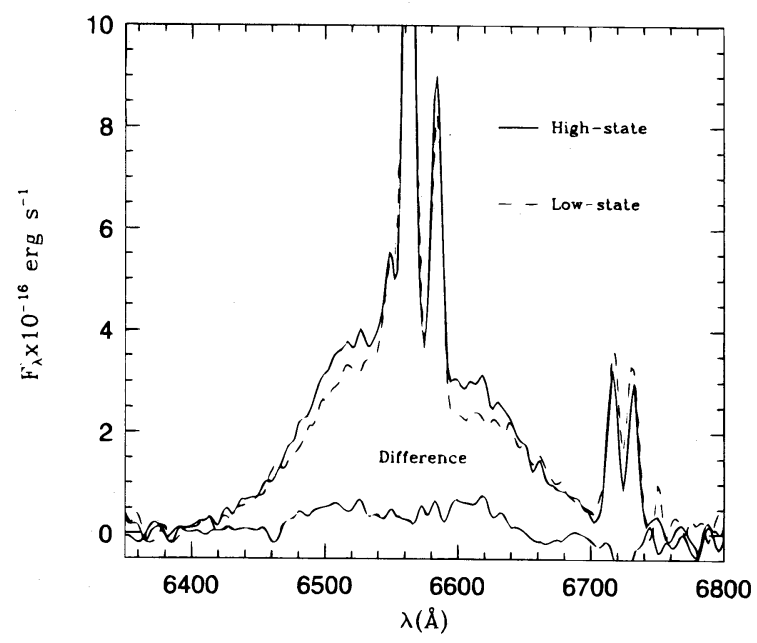

Fig. 3.-Spectra in the $\mathrm{H} \alpha,[\mathrm{N}$ II], [S II] region for the high and low states. The difference spectrum is displayed at the bottom. The variable $\mathrm{H} \alpha$ line shows a flat-topped symmetrical profile with FWHM $\sim 6000 \mathrm{~km} \mathrm{~s}^{-1}$ and quite unconspicuous wings. All spectra are plotted to scale, without any vertical displacement. 
best fit was obtained for a disk viewed at an inclination angle $i=33^{\circ} \pm 1^{\circ}$ with inner and outer radii $\xi_{1}=1500$ \pm 100 and $\xi_{2}=7000 \pm 100$, respectively (in units of $G M / c^{2}$, where $M$ is the mass of the black hole). The line emissivity of the disk follows the law $\xi^{-q}$ with $q=2.0 \pm 0.1$. Local broadening, possibly due to electron scattering or turbulent motions, was introduced, being the broadening represented by a Gaussian profile of width $\sigma=1600 \pm 50$ $\mathrm{km} \mathrm{s}^{-1}$ (in the rest frame of the emitter). The errors in the fit were determined by varying the parameters until the fit did not adequately reproduce the observed profile. Figure 4 shows the $\mathrm{H} \alpha$ line together with the best-fit disk model. It can be seen that the model reproduces adequately the overall characteristics of the broad profile. There is a small additional component at the blue side of the narrow $\mathrm{H} \alpha$ line that the disk model did not account for, which we attribute to emission that originates in the narrow-line or intermediate-line region of the galaxy and is therefore physically distinct from the accretion disk. The residual spectrum, including the $[\mathrm{N} \mathrm{III}] \lambda \lambda 6548,6584$ and $\mathrm{H} \alpha$ narrow lines, is shown in the bottom panel of Figure 4.

Based on the above fit, we propose a model for C16.16 in which a circular, relativistic Keplerian disk around a black hole exists at the center of this galaxy. According to the model, a quasi-spherical, hot ion torus occupies the inner disk $\left(\xi \leq \xi_{1}\right)$ and illuminates the thin outer disk with ionizing radiation, driving the line emission (Chen \& Halpern 1989). The ion torus may be indirectly responsible for the large contribution of starlight to the optical continuum and the relatively strong low-ionization lines, although the value of the ratio between the line fluxes [O I] $\lambda 6300 /[\mathrm{O}$ III] $\lambda 5007$, equal to 0.07 , is smaller than the median value $(0.14)$ shown by disklike emitters (Eracleous \& Halpern 1994).

There also exist other routes that can lead to the emission of double-peaked profiles, as in the case of a circular disk with local inhomogeneities (hot spots) in dissipation due to

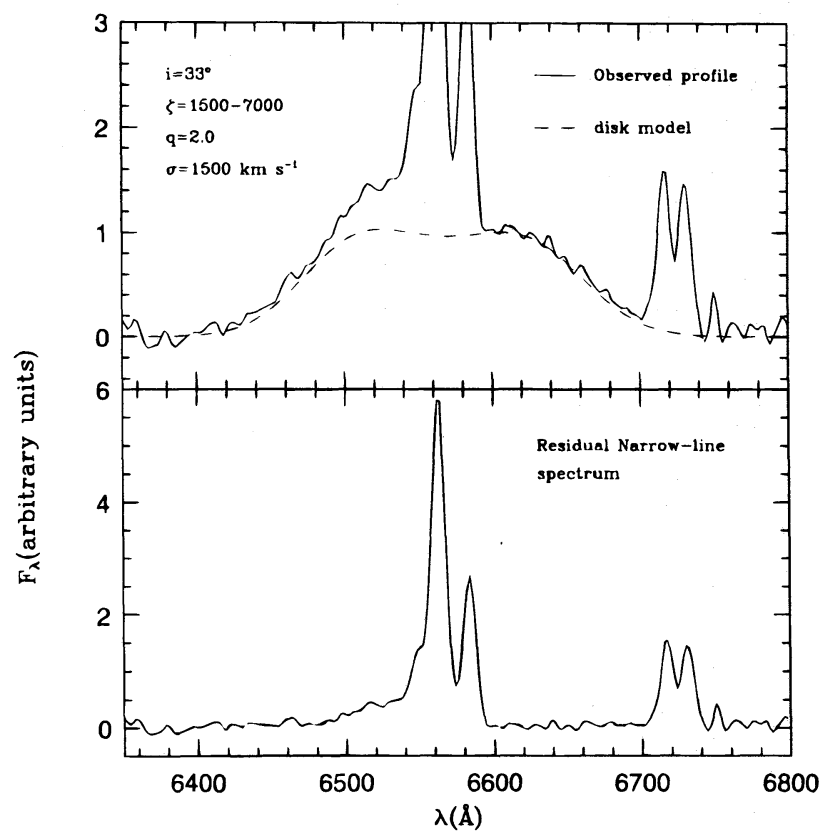

FIG. 4.-Top: Fit of the circular relativistic Keplerian disk model to the observed double-peaked $\mathrm{H} \alpha$ profile after continuum subtraction. Bottom: Residual spectrum resulting from the subtraction of the model profile from the observed profile. the Keplerian shear. The dissipation time for such inhomogeneities can vary between several months and a few decades, depending on the mass of the central black hole. Also, spiral waves on the line profiles from a circular disk (Chakrabarti \& Wiita 1993, 1994) could provide profiles that have the blue peak stronger than the red peak or vice versa, depending on the orientation of the spiral pattern and the number of spiral arms. As the pattern precesses, it causes the profiles to vary, with the possibility of the two peaks' alternating in strength. In this case, changes in the profile can be detected in time intervals of a few years.

We believe that only extensive monitoring of C16.16's Balmer line profiles can allow one to determine or at least constrain which of the above scenarios can be correctly applicable to this galaxy. If double-peaked profiles are generated in an accretion disk, one would expect to see very rapid profile variability with a timescale of the order of the light crossing time for the accretion disk. Such a variation was not detected in C16.16 for the two available snapshots, since we have assumed that the variable part of the line comes from the intermediate Balmer continuum rather than from the disk, but it may also indicate that the profile could have varied and then returned to its initial state. As a first step, it would be very helpful to determine whether C16.16 is a radio galaxy. This can be done directly by radio observations or indirectly by deep imaging to identify the morphology of the host galaxy. It would also help to observe this galaxy in other spectral regions, especially in the UV and X-ray bands. With the present evidence we can neither rule out nor accept any of the above hypotheses because the evidence is not conclusive.

\section{CONCLUSIONS}

The main conclusions of this spectral study of the faint, distant Seyfert 1 galaxy C16.16 can be summarized as follows:

1. The underlying stellar population in the sampled region $(\sim 3 \mathrm{kpc} \times \sim 13.5 \mathrm{kpc})$ of $\mathrm{C} 16.16$ has a strong contribution $(24 \%)$ of stellar components with ages $t \leq 500 \mathrm{Myr}$. In the low-state spectrum, at $5870 \AA, 90 \%$ of the continuum flux arises from this component while at high activity this contribution decreases to $70 \%$. In the near-UV, at $3200 \AA$ the underlying galaxy contributes $85 \%$ and $34 \%$, respectively. This result reflects the strong variation of the AGN continuum between the 1992 October and 1994 August observations.

2. The overall nonstellar continuum of the $\mathrm{C} 16.16 \mathrm{nu}$ cleus between 3200 and $6800 \AA$ is described in the high-state spectrum by a combination of a power-law spectrum, Balmer continuum emission, $\mathrm{Fe}$ II emission multiplets, and the merging of high-order Balmer lines. In the low-state spectrum, only the $\mathrm{Fe}$ II component was found. The remaining components, if any, are reduced to the noise level.

3. The narrow-line region shows a physical structure very similar to that normally found in other Seyfert galaxies. From photoionization models, we find an ionization parameter $U=10^{-2.8}$, electron density $N_{e}=10^{3.2} \mathrm{~cm}^{-3}$, electron temperature $T_{e}=12,500 \mathrm{~K}$, and solar abundances except for nitrogen, which was half-solar. The lowionization lines show large equivalent widths, a property that seems to be common in double-peaked AGNs.

4. The broad and asymmetric Balmer lines show a conspicuous double-peaked structure, well reproduced by the emission of a circular relativistic Keplerian disk model. In 
this context, the presence of an ion torus in the inner disk would illuminate the outer disk with ionizing radiation, driving the line emission. The disk profile seems to respond to continuum changes with a different time delay than the intermediate-width component of the line and the near-UV excess, suggesting that they are either generated in different spatial regions or by different processes.

5. Although some observational evidence suggests that C16.16 is a radio galaxy (i.e., moderate luminosity, large stellar population, and broad and double-peaked Balmer lines) other evidence contradicts this: narrow-line ratios such as $[\mathrm{O} \mathrm{II}] /[\mathrm{O} \mathrm{III}]$ and $[\mathrm{O} \mathrm{I}] /[\mathrm{O} \mathrm{III}]$ quite typical of Seyfert galaxies; Balmer decrement very close to case B instead of the higher ratios expected in radio galaxies. So, whether $\mathrm{C} 16.16$ is a radio galaxy or not remains as an open question. Deep optical imaging to resolve the morphology of the host galaxy would provide a very good clue on this issue, although radio observations would provide the definite answer.

We thank Beverly Wills for kindly providing the Fe II and Balmer continuum templates. We also thank Michael Eracleous for valuable comments, which helped improve this manuscript. A. R. A. would like to thank Claudia Winge and Charles Bonatto for much support and advice during the preparation of this work. This work has been partially supported by the Brazilian Research Council CNPq to A. R. A. and by the Chilean institution FONDECYT (Fondo Nacional de Ciencias) under grant 92-1083 to J. M.
Alloin, D., Poisson, C., \& Pelat, D. 1988, A\&A, 200, 17

Bica, E. 1988, A\&AS, 195, 76

Bica, E., \& Alloin, D. 1986, A\&A, 162, 21

Bica, E., Alloin, D., \& Schmitt, H. 1994, A\&A, 283, 805

Burstein, D., \& Heiles, C. 1984, ApJS, 54, 33

Cassidy, I., \& Raine, D. J. 1993, MNRAS, 260, 385

Chakrabarti, S., \& Wiita, P. J. 1993, A\&A, 271, 216 1994, ApJ, 434, 518

Chen, K., \& Halpern, J. P. 1989, ApJ, 334, 115

Chen, K., Halpern, J. P., \& Filippenko, A. V. 1989, ApJ, 339, 742

Collin-Souffrin, S. 1987, A\&A, 179, 60

Dumont, A. M., \& Collin-Souffrin, S. 1990, A\&A, 229, 313

Eracleous, M. \& Halpern, J. P. 1994, ApJS, 90, 1

Evans, I. N. 1988, ApJS, 67, 373

Ferland, G. J. 1989, Ohio State Univ. Internal Rep. 89-001

Gaskell, C. M. 1988, in Lecture Notes in Physics, 307, Active Galactic

Nuclei, ed. H. R. Miller \& P. J. Wiita (Berlin: Springer), 61

Korista, K. T. 1992, ApJS, 79, 285

Mathews, W. G., \& Ferland, G. J. 1987, ApJ, 323, 456

\section{REFERENCES}

Maza, J., Ruiz, M. T., González, L. E., \& Wischnjewsky, M. 1989, ApJS, 69, 349

1992 Rev. Mexicana Astron. Astrofis., 24,147

Maza, J., Ruiz, M. T., González, L. E., Wischnjewsky, M., \& Antezana, R. 1994, Rev. Mexicana Astron. Astrofis., 28, 187

McCall, M. L. 1984, MNRAS, 208, 253

Osterbrock, D. E. 1989, Astrophysics of Gaseous Nebulae and Active Galactic Nuclei (Mill Valley, CA: Univ. Sci.)

Osterbrock, D. E., Koski, A. T., \& Phillips, M. M. 1976, ApJ, 206, 898

Peterson, B. M., et al. 1991, ApJ, 368, 119

Reichert, G. A., et al. 1994, ApJ, 425, 582

Rokaki, E., Boisson, C., \& Collin-Souffrin, S. 1992, A\&A, 253, 57

Stirpe, G. M., de Bruyn, A. G., \& van Groningen, E. 1988, A\&A, 200, 9

Stirpe, G. M., et al. 1994, ApJ, 425, 609

Wamsteker, W., et al. 1990, ApJ, 354, 446

Wills, B., Netzer, H., \& Wills, D. 1985, ApJ, 288, 94

Zheng, W., Binette, L., \& Sulentic, J. W. 1990, ApJ, 365, 115

Zheng, W., Veilleux, S., \& Grandi, S. A. 1991, ApJ, 381, 418 